\title{
AN EXAMPLE OF STATUS MARMORATUS OF THE CEREBRAL CORTEX
}

BY

\author{
R. M. NORMAN
}

From Stoke Park Colony

(RECEIVED 9TH JULY, 1937)

THE phenomenon of hypermyelinization has received much attention from neuropathologists since C. Vogt's (1911) original description of status marmoratus of the corpus striatum. It is now well established that hypermyelinized fibres in the form of plaques or as the peculiar networks which first suggested the name " marbled" may be present in many parts of the nervous system, though they are most common in the basal ganglia and cerebral cortex. A review of the recorded cases shows that several æatiological factors may be operative in the production of this remarkable state (Hallervorden, 1930). Thus, hypermyelinized plaques have been found associated with traumatic lesions (Freund and Vogt, 1911; Pfeiffer, 1925; Scholz, 1924), general paralysis (Bielschowsky, 1924), disseminate sclerosis (Wertham, 1924), von Recklinghausen's disease (Bielschowsky, 1924), senile dementia (Stief, 1924), telangiectiasis of the cortex (Bielschowsky, 1924), carbon monoxide poisoning (Spatz, 1931), febrile illness in infancy (Anton, 1896 ; Scholz, 1924 ; Case, 1934 ; Lowenberg and Malamud, 1933), and birth injury (Meyer and Cook, 1936). Familial instances of status marmoratus of the corpus striatum are also on record (Anton, 1896 ; Scholz, 1924 ; Vogt, 1920). The pathogenesis of the condition is obscure. Since hypermyelinization is almost invariably accompanied by some degree of gliosis, there are grounds for believing that a perversion of glial function has led to the abnormal myelin production. Considerable uncertainty, however, still exists as to the mode of origin of the abundant axis cylinders found in the plaques. Shrinkage of the tissues might conceivably produce the linear hypermyelinized scars so frequently seen in the cerebral cortex, but it is difficult to imagine how such a mechanism could produce the distinctive networks of a true status marmoratus. Bielschowsky's (1924) suggestion that previously naked axis cylinders have become myelinized also fails to explain the excessive number and peculiar arrangement of the fibres. Most authors seem to have followed Scholz (1924) in interpreting the abnormal axis cylinder development as a regenerative phenomenon, despite the fact that in experimental animals only ephemeral and abortive regenerative activity has been observed. The experiments of Borst (1904) have been cited as proving that a new growth of myelinized fibres can actually occur after cerebral lesions, but, as Cajal (1928) has pointed out, his findings are open to other interpretations and he did not employ neurofibrillar staining methods.

I have thought the following case worthy of record since, among other points of interest, it exemplifies the association of status marmoratus with certain cortical abnormalities of prenatal origin.

\section{Personal Case}

The patient, a male aged at death 27 years and 2 months, died at Stoke Park Colony on 18th April, 1935, from Banti's disease after an illness of approximately 
sixteen months' duration. He was an illegitimate child and nothing is known of his life prior to admission to the Colony. Examined in 1928, he was classified as an idiot having a mental age less than 3 years. The records state that he was unfit for any but the simplest occupation, was dirty in habits, and was very excitable and inclined to be dangerous to others. He suffered from grand mal, was " partially blind," his speech was limited and indistinct and some weakness was observed in the legs. The Wasserman reaction in the blood was negative (November, 1934).

A neurological examination made shortly before death gave the following information. The pupils reacted to light and on convergence. The tendon reflexes in the upper limbs were very brisk and equal. The upper abdominal reflexes were not obtained. The lower abdominal reflexes were present and equal. The cremasteric reflexes were absent. In the lower limbs, the knee-jerks were equal and exaggerated, Strümpel's sign being positive. The ankle-jerks were equal and increased. There was no knee or ankle clonus. The plantar reflexes were flexor. There was no record at any time of athetoid movements.

\section{Pathological Examination}

At the autopsy which was made 16 hours after death the diagnosis of Banti's disease was confirmed. At the time of removal of the brain no abnormal adhesions between the dura mater and the calvaria were found and there was no sign of old sinus thrombosis. The meninges appeared to be normal.

The brain was markedly micrencephalic. Measurements obtained from the right hemisphere gave the following readings : length, $140 \mathrm{~mm}$. ; breadth, $54 \mathrm{~mm}$. ; height, $100 \mathrm{~mm}$. A comparison between this brain and the normal brains in the collection at Stoke Park Colony showed that its size corresponded approximately to that of an infant of a year old. This reduction in total volume was in large measure due to the especially small size of the parietal and occipital lobes. After stripping the leptomeninges, it was seen that both hemispheres exhibited gross structural abnormalities. On both sides the Rolandic sulcus was laid down in two parts, and in the left hemisphere the upper part of the postcentral gyrus seemed to be represented by a thin, ribbon-like structure which was concealed in the depth of the central sulcus. This peculiar formation took its origin from the posterior wall of the precentral gyrus at a point above the general level of the floor of the Rolandic sulcus. The presence of numerous small gyri interspersed between gyri of more normal size gave the upper part of the parietal lobe a markedly abnormal appearance. The mesial surface and the upper part of the outer surface of the occipital lobes were similarly affected. The calcarine fissures could be identified, but the arrangement of the neighbouring gyri was confused by an over-production of small, shrunken, and apparently sclerotic convolutions. These irregularities were only approximately symmetrical in the two hemispheres. A more detailed examination of these changes gave the impression that while some of the appearances presented by the larger gyri might be attributed to the distorting influence of sclerosis (ulegyria), this factor could not account for all the abnormalities found in the convolutional pattern. A malformation of the cortex in the form of a partial microgyria seemed to have formed the basis of this complex arrangement.

On sectioning the brain it was seen that the white matter of the centrum ovale was very thin subjacent to the abnormal portions of the cortex, the lateral ventricle in this position being dilated. Many of the gyri presented a constriction in their basal portions. The brain tissue in such situations was extremely tough and evidently sclerosed. In the less sclerotic gyri, partial shrinkage had produced an effect somewhat resembling candle gutterings.

In the dorsal part of the putamen an abnormal network of white fibres characteristic of status marmoratus could be distinguished macroscopically. The corpus callosum 
was noticeably small and at a point just anterior to the splenium narrowed to a depth of about $2 \mathrm{~mm}$.

Cerebral Cortex.-The abnormal construction of the small and distorted gyri in the parieto-occipital part of the cortex may most conveniently be explained by reference to the illustrations. Fig. 1 shows typical gyri with a variety of myelin abnormalities, the following points being especially noteworthy :

(a) The occurrence of several small gyri developed upon one parent stem (see also Fig. 3).

(b) Typical retiform status marmoratus in the larger gyri.

(c) Diffuse hypermyelinization in three small convolutions $a, b, c$ (Fig. 8). The myelinated fibres in these areas (as also in Fig. 6) were present in dense whorls and practically confined to the grey matter, the central core of the gyrus containing very few axis cylinders. The latter fact was confirmed in Bielschowsky preparations. The molecular layer in such gyri showed an abnormal wealth of myelinated fibres.

Sections stained for glial fibres by Holzer's method showed that the parts of the cortex where myelin was scanty or absent, more particularly the constricted basal parts of the gyri, were the seat of an extremely dense sclerosis. Small areas of rarefaction and porus formation were frequently seen (Fig. 2). The blood vessels in such areas showed intense fibrotic thickening (Fig. 11). The myelinated parts of the central cores showed a fairly dense isomorphous gliosis. In the areas of the grey matter exhibiting hypermyelinization a much finer gliosis was found, which was usually only demonstrated with difficulty (Fig. 14) and which was often invisible under low-power magnification. In some areas of status marmoratus repeated staining of sections failed to demonstrate any glial fibres in the grey matter, although a dense marginal gliosis was usually observed together with obvious proliferation of glial fibres in the central core of white matter.

An interesting glial abnormality was found in sections taken from the macroscopically normal temporal pole. Special staining showed the presence of numerous very large fibre-forming astrocytes in the grey matter of the cortex, chiefly in the lower layers (Fig. 13). Other abnormal glia cells were characterized by their coarse, fleshy processes and large, deeply-staining cell bodies. Figs. 3 and 4 illustrate many of the peculiarities described above, and in addition, a remarkable abnormality in the arrangement of the myelinated axons composing the central parent stem of a cluster of small gyri. The fibres derived from these gyri are present as two strands on either side of a central area devoid of myelin. The glial picture is practically the reverse of the myelin preparation.

Sections of the cortex stained for nerve cells showed a variety of abnormalities. The most common finding in the area showing status marmoratus was a patchy distribution of the cytons. In some of the smaller gyri, for example, those in which diffuse hypermyelinization was present, the nerve cells were arranged in clusters and columns with no attempt at differentiated lamination (Fig. 7). The majority of these cells were well-formed pyramidal cytons, some being of disproportionately large size compared with their fellows. Bielschowsky's staining revealed a very rich axis cylinder development confined to the grey matter. The impression given by such gyri was not that of a normal cortex which had undergone pathological change, but rather that of a malformation. In contrast to this latter type of gyrus, those parts of the cortex in which a severe degree of sclerosis was present showed a nerve-cell picture typical of ulegyria (the "granular atrophy" of Spatz). Such changes were most frequently encountered in the walls and basal portions of the gyri, the line of demarcation between the atrophic part of the gyrus and the bulbous neurone-bearing crown being often abrupt. In the upper part of the Rolandic motor area there was a typical status marmoratus (Fig. 5). Sections taken from this area and stained for nerve cells showed that the deepest layer of the cortex (L.VI) was in parts entirely absent, its place being taken by proliferated neuroglia. The Betz cells of L.V thus 
represented the lowest recognizable limit of the grey matter (Fig. 9). These cells were numerous and most were of fully adult size. Some displayed a marked degree of apolarity and many had assumed a circular or ovoid shape, the nucleus being displaced in a lateral direction. Sections stained by Bielschowsky's method showed that the parts of the cortex exhibiting status marmoratus also showed a dense network of fine axis cylinders. One could distinguish several examples of Betz cells possessing numerous short processes. In favourable sections these latter could be shown to break up into finer branches contributing to the surrounding rich fibrillary network of the status marmoratus (Fig. 12). In the small ribbon-like upper part of the postcentral gyrus the nerve cells were confined to the small bulbous crown of the convolution, the remainder of the gyrus being composed of dense glial fibres and containing few myelinated fibres. A much finer gliosis was present in the neuronebearing area where a diffuse hypermyelinization of fibres was observed (Fig. 14).

Two other abnormalities were encountered which are worthy of mention. One of these is shown in Fig. 10, a low-power view of the visuosensory cortex. The lower layers of the cortex were spread out into two thin bands that contrasted markedly with the denser aggregation of nerve cells in the outer part of the gyrus, from which they were separated by an appreciable depth of tissue containing only glial nuclei. The other abnormality was met with in one block of tissue removed from the macroscopically abnormal parieto-occipital region. This was the presence of a heterotopic collection of some 20 abnormally shaped nerve cells lying in the white matter at a point approxinately half-way between the ependyma and the small sclerotic cortical gyri. This heterotopia was sufficiently large to show as a pale area in myelin preparations.

An examination of cortical areas remote from those displaying the gross abnormalities recorded above showed little to distinguish them from those commonly encountered in cases of low-grade mental deficiency. From the point of view of size, the nerve cells of the supragranular layers of the temporal and frontal lobes were moderately well developed, but their arrangement was in general typically irregular. No defect was found in the pyramidal cell layer of the cornu ammonis.

The microglia in the cortex showed no abnormality.

The oligodendrocytes in the areas of status marmoratus showed acute swelling.

Cerebellum.-The only abnormality found in sections taken from the vermis consisted in the occasional presence of "torpedoes" in the axons of the Purkinje cells. There was a slight gliosis of the white matter of the folia.

Putamen.-The presence of a well-marked status marmoratus which had been observed macroscopically in the dorsal part of the putamen was confirmed when sections were stained for myelin (Fig. 15). Bielschowsky's method showed in the areas of hypermyelinization a corresponding number of fine axis cylinders. As in the cortex, no bulbs, spirals, or other phenomena suggestive of regeneration were seen. There was a fine gliosis in these areas, which was demonstrated only after repeated efforts with the Holzer stain. Corresponding pictures of myelin and glia were not obtained. A diminution in the numbers of ganglion cells was observed and an increase in small glial nuclei, many of which proved to be those of swollen oligodendrocytes. The microglia showed no abnormality, nor were the blood vessels increased in number. The external capsule showed an intense gliosis and was densely myelinated. The claustrum and some parts of the insular cortex also showed areas of hypermyelinization.

Thalamus.-No status marmoratus was found in the thalamus. The subependymal region showed an increase of fibrous neuroglia. The glial fibrosis seemed to be densest in the neighbourhood of the terminal vein.

Head of the Caudate Nucleus, Globus Pallidus, Midbrain, Pons, Medulla, and Spinal Cord.-No abnormality in myelin or nerve cells was found in these parts of the nervous system.

External Geniculate Body.-It was about one-third the size of a normal control 
sectioned in a similar manner for purpose of comparison. Its cell content was also notably inferior to the normal. The characteristic structure of the nucleus could, however, be distinguished.

\section{Discussion}

The foregoing microscopical examination has shown that this case is one of considerable complexity, and it is by no means easy to trace the temporal sequence of the various abnormalities described. There are several indications, however, that some of the cortical changes had resulted, in the first instance, from a disturbance of development occurring during fotal life. In contrast to the usual case of ulegyria in which previously normal convolutions have become shrunken and distorted by sclerosis, the fissuring of this brain was abnormally complex owing to the presence of a multiplicity of small gyri. The microscopical examination of such areas afforded many instances of gyri the abnormal cytoarchitectonics of which could not be ascribed simply to destructive processes. It would therefore seem justifiable to regard such gyri as primarily microgyric. The presence of a heterotopic collection of nerve cells in the subcortical white matter strengthens this supposition. This picture of malformation, however, was complicated by widespread evidence of degenerative process, involving not only microgyric areas but larger and more adult convolutions also. These atrophic changes were most marked in the walls and basal portions of the gyri, leaving the bulbous, neurone-bearing crowns relatively intact. This regional distribution is not uncommon in examples of atrophic lobar sclerosis without status marmoratus, and is probably due to anatomical factors of vascular supply. The findings in the present case thus seem to admit of two different interpretations : the first, that a pathological process leading to sclerosis has been engrafted upon a pre-existing malformation ; the second, that both the appearances suggestive of malformation and the atrophic changes have arisen as the result of a pathological process acting upon the cerebral cortex during an early stage of its development. The latter explanation has the merit of accounting for the observed facts without involving a double pathology.

The main interest of this case lies in the widespread hypermyelinization present in the cerebral cortex. As has been pointed out in the introduction, the most difficult problem raised by a study of status marmoratus is not the mechanism by which the axis cylinders have become myelinated but the origin of the axis cylinders themselves. As in the striatum, so in the cerebral cortex one is confronted with the paradoxical finding of an excessive production of myelinated fibres together with a diminution of the total nerve-cell content of the area involved in the change. In the present case the axis cylinders in the areas of status marmoratus showed none of the bulbs, spirals, or spools that some investigators consider to be the result of regenerative processes. There is another possible explanation as to the origin of these abnormal strands and networks of axis cylinders which seems to have escaped the notice of previous observers. The fibres may have arisen not as the result of true regeneration but by a process analogous to that described by Cajal (1928) as compensatory collateral hypertrophy. In Cajal's experiments section of the axon of pyramidal cell distal to the origin of the initial collaterals resulted in the compensatory hypertrophy of these collateral branches. This reaction, which was named " arciform transformation" of the nerve cell, led to the development of a neurone which was " a perfect reproduction of a cell with a short axon except that the terminal arborization is composed of hypertrophic branches which involve an enormous part of the cortex." It is not without significance that those gyri showing the densest marbled state were those whose basal portions were affected by the most severe sclerosis and contained only a small number of axis cylinders. The hypermyelinization of the axis cylinders in the areas of status marmoratus may be attributed, as in other cases, to a perversion of glial activity. It was usually possible to demonstrate a slight excess of glial fibres 
in these areas of the grey matter, a " partial sclerosis" in the sense of Scholz being sometimes present. The failure to find gliosis in some of the marbled areas may be due to technical faults, though it may be pointed out that Case (1934) and in one instance C. Vogt (1925) failed to find a glial proliferation corresponding to the myelin networks. Possibly the fact mentioned by Meyer (1936), that the degree of marbling tends to vary from section to section, may explain this discrepancy. Nevertheless, in the present case it would be inadvisable on the histological findings to speak of " myelinated scars" being present in the grey matter, though the white matter showed numerous examples of such formations.

From the clinical standpoint this case of status marmoratus is remarkable in that the patient did not exhibit during life the athetoid movements characteristic of this form of striatal abnormality. The explanation is probably to be found in the coincident involvement of the cerebral cortex, more particularly of the lower layers of the precentral gyrus, the integrity of which is a necessary factor in the exteriorization of complex hyperkinetic movements.

\section{Summary}

The pathological examination of the brain of an adult epileptic idiot showed extensive status marmoratus of the cerebral cortex and of the dorsal portion of the putamen.

The parietal and occipital lobes were fissured in an abnormally complex manner, and showed what was considered to be a combination of malformation and atrophic changes.

A short account of some of the problems of the pathogenesis of status marmoratus has been given. The suggestion is made that the wealth of axis cylinders present in some of the marbled areas in the present case owed their origin not to a true regenerative activity but to a process akin to the compensatory collateral hypertrophy of Cajal.

It is a pleasure to record my thanks to Professor Berry for permission to publish these notes and also to Dr. A. Meyer for his helpful interest in the pathological findings of this case. This work was carried out on behalf of the Burden Mental Research Trust.

\section{REFERENCES}

Anton, G. (1895). Jahrb. Psychiat., 14, 141.

Bielschowsky, M. (1924). J. Psychol. Neurol., Lpz., 31, 125.

Borst, M. (1904). Beitr. path. Anat., 36, 1.

Cajal, S. Ramon y (1928). Degeneration and Regeneration of the Nervous System. Vol. 2. London.

Case, T. J. (1934). Arch. Neurol. Psychiat., Chicago, 31, 817-23.

Freund, C. S., and Vogt, C. (1911). J. Psychol. Neurol., Lpz., 18, 4, 489.

Hallervorden, J. (1930). "Die Extrapyramidalen Erkrankungen" : Bumke's Handbuch der Geisteskrankheiten, p. 1038.

Lowenberg, K., and Malamud, W. (1933). Arch. Neurol. Psychiat., Chicago, 29, 104.

Meyer, A., and Cook, L. C. (1936). J. Neurol. Psychopath., 16, 341.

Pfeiffer, F. (1925). Arch. Psychiat., 72, 728.

Scholz, W. (1924). Z. ges. Neurol. Psychiat., 88, 355.

Spatz, H. (1931). Verh. Ges. dtsch. Nervenärtz, 4, 531.

Stief, A. (1924). Z. ges. Neurol Psychiat., 91, 579.

Vogt, C. (1911). J. Psychol. Neurol., Lpz., 18, 4, 479.

(1920). Ibid., 25, 279.

-, C. and O. (1920). Ibid., 25, 627.

Wertham, F. and F. (1934). The Brain as an Organ, p. $336 . \quad$ London. 

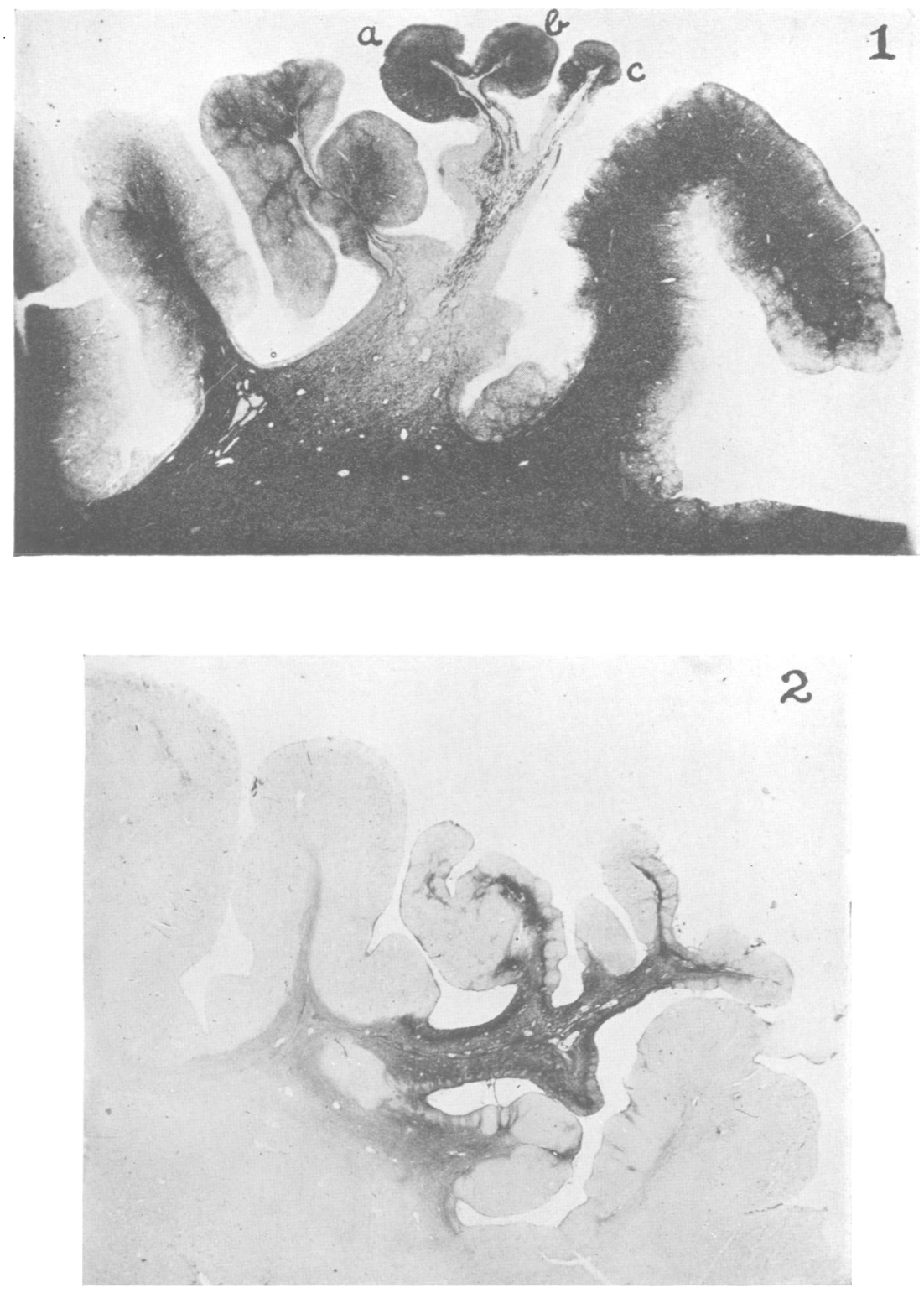

Fig. 1.-Gyri from the occipital region. The small gyri $a, b, c$ show dense whorls of hypermyelinated fibres practically confined to the grey matter and molecular layer of the cortex. Anderson's modification of the Kulschitsky-Pal method; $\times 5 \frac{3}{4}$.

Fig. 2.- Section taken from the same block of tissue as shown in Fig. 1. Holzer's stain ; $\times 5 \frac{3}{4}$. 

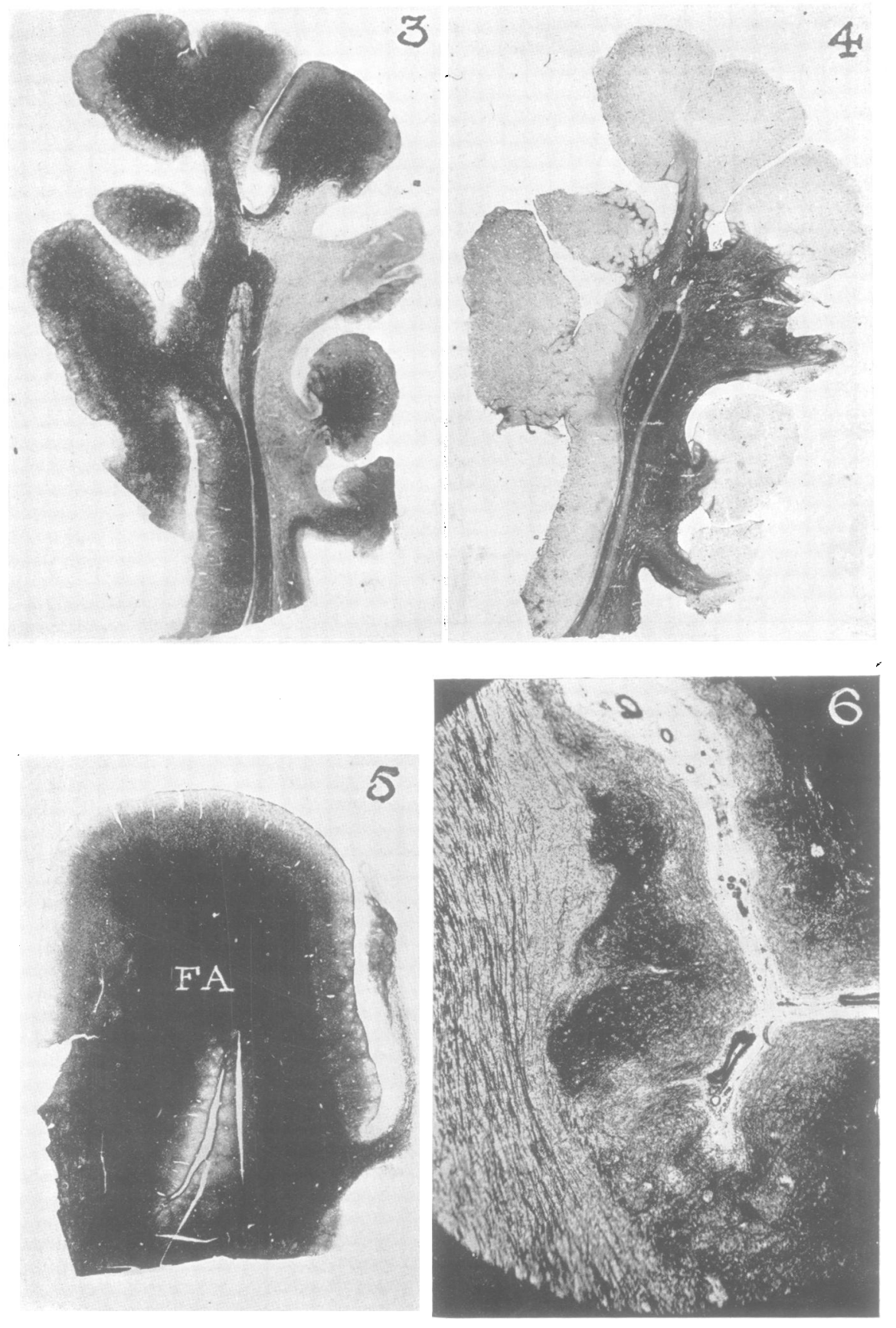

Fig. 3.-Gyri from the occipital lobe. Kulschitsky-Pal stain ; $\times 4$.

Fig. 4. - Section taken from the same block of tissue as shown in Fig. 3. Holzer's stain; $\times 4$.

Fig. 5.-Upper precentral gyrus (FA) showing status marmoratus in the cortex adjacent to the small atrophic postcentral gyrus. Kulschitsky-Pal stain ; $\times 3$.

Fig. 6.-Diffuse hypermyelinization of the cortex ; the fibres are largely confined to the grey matter. Kulschitsky-Pal stain; $\times 13$. 

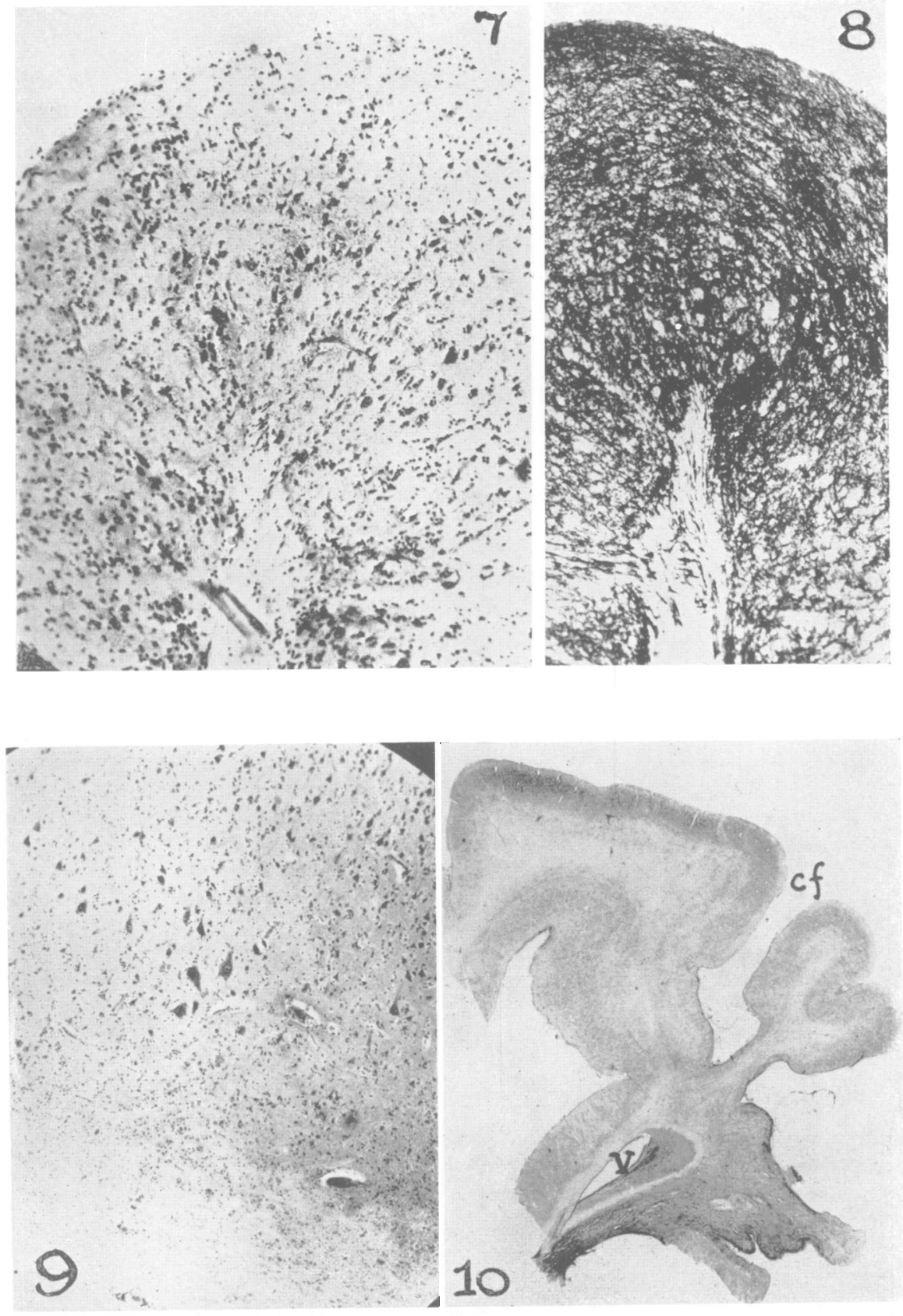

Fig. 7.-Nerve-cell picture of gyrus $b$ in Fig. 1. Cresyl violet ; $\times 60$.

Fig. 8.-As in Fig. 7. Kulschitsky-Pal stain, $\times 60$.

Fig. 9.- Nerve-cell picture of motor cortex showing a polarity of Betz cells and absence of the sixth layer.

Fig. 10.-Low-power view of calcarine fissure showing abnormal arrangement of the deeper layers of the visuosensory area, typical basal constriction of the gyrus, and thinness of the subcortical white matter. $v=$ ventricle. Cresyl violet; $\times 4$. 

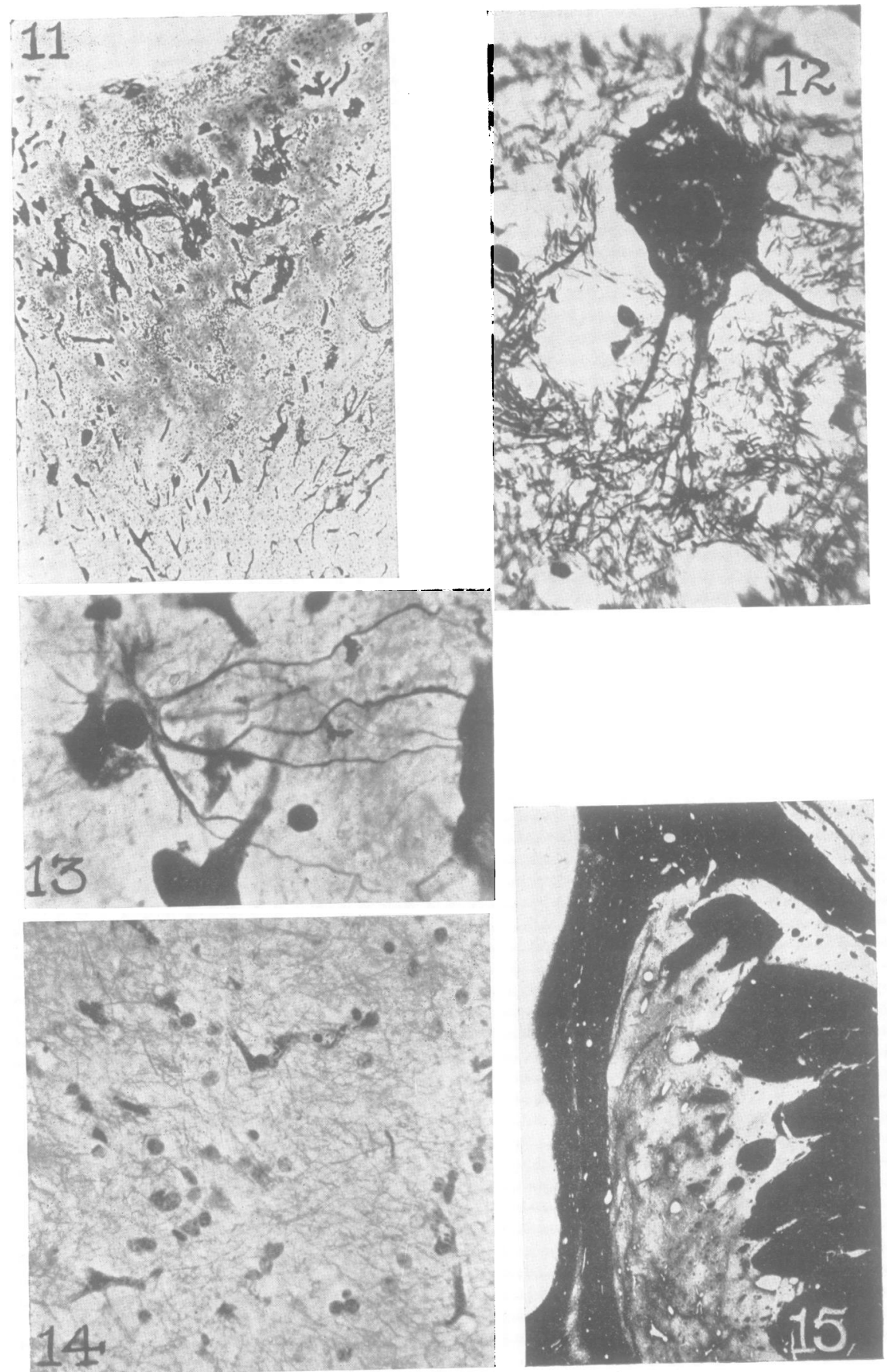

Fig. 11.-Fibrotic vessels in an area of severe sclerosis. Perdrau's stain; $\times 36$. Fig. 12. - Betz cell with branching process contributıng to the axis cylinder network of an area of status marmoratus. Bielschowsky's stain : $\times 600$.

Fig. 13.-Typical hypertrophic fibre-forming astrocytes found in sections from the temporal pole. Hortega's method for astrocytes ; $\times 600$.

Fig. 14.-Type of fine gliosis found in areas showing hypermyelinisation. Holzer's stain; $\times 270$.

Fig. 15.-Status marmoratus of the putamen. Kulschitsky-Pal stain; $\times 4$. (The section has been rather over-differentiated in order to show up clearly the marbled appearance of the putamen. Consequently, the cortex of the insula seems devoid of fibres.) The claustrum also shows hypermyelinization. 